\title{
IL CONSOLIDAMENTO DEL TIBURIO DEL DUOMO NEGLI ANNI SETTANTA DEL XX SECOLO
}

\author{
LEONE CORRADI DELL'ACQUA (*)
}

SunTo. - Negli anni sessanta del secolo scorso il Duomo di Milano ha evidenziato uno stato di sofferenza che si è andato rapidamente aggravando fino a divenire decisamente preoccupante. Per ripristinare la sicurezza e l'agibilità del monumento si è reso necessario un intervento di consolidamento molto delicato, alla cui definizione e realizzazione alcuni docenti del Politecnico di Milano hanno dato un contributo determinante. In questa nota si riassumono gli aspetti salienti della vicenda.

$* * *$

ABSTRACT. - At about 1960, some signs of damage became evident in Milan's cathedral, which rapidly increased to reach a very serious situation. Restoring the safety and the functionality of the monument required a rather difficult operation, which was designed and carried out with the essential contribution of a number of experts from the Politecnico di Milano. In this paper the main aspects of this sequence of events are summarized.

Nel 1961 l'ing. Carlo Ferrari da Passano, da poco assunto alla Veneranda Fabbrica del Duomo di Milano come direttore tecnico, ha osservato presenza di piccole ma diffuse lesioni, indice di uno stato di sofferenza del monumento. La situazione si è andata rapidamente aggravando, con la formazione di nuove lesioni e l'accrescimento di quelle esistenti, al punto che in pochi anni le condizioni in cui si trovava la cattedrale erano divenute preoccupanti e alla fine degli anni sessanta decisamente drammatiche. La Fig. 1, che mostra le lesioni rinvenute in uno dei pilastri che sostengono il tiburio all'inizio del 1969, illustra più di molte parole la gravità della situazione.

(*) Istituto Lombardo Accademia di Scienze e Lettere, Politecnico di Milano, Italia. E-mail: leonemaria.corradidellacqua@fastwebnet.it 
Il Duomo di Milano, come del resto analoghi monumenti dell'epoca, è una struttura di per sé "fragile", che presenta margini di sicurezza molto ridotti. Le sue vicende costruttive sono state piuttosto travagliate e non sempre guidate da scelte felici. Già nei primi anni della sua costruzione sono state sollevate alcune perplessità, segnatamente sull'adeguatezza delle fondazioni dei pilastri, che sono state oggetto di accesi dibattiti ma che alla fine non sono state prese in seria considerazione. Un'ulteriore fonte di debolezza è stata introdotta con la costruzione della cupola e del tiburio alla fine del quindicesimo secolo. Per salvaguardare l'unità architettonica del monumento, si sono voluti conservare i preesistenti archi gotici, pur se insufficienti a reggere il carico aggiuntivo. Questo è stato affidato ad archi a tutto sesto, non visibili, che agiscono eccentricamente sui pilastri, aggravandone la spinta verso l'esterno (le catene introdotte per fronteggiare il problema sono entrate quasi immediatamente in crisi). Inoltre, nella seconda metà del diciottesimo secolo è stata innalzata la guglia maggiore, la cui presenza comporta un considerevole peso aggiuntivo che grava sulla sommità della cupola e si scarica (eccentricamente) sui pilastri che la sorreggono. La

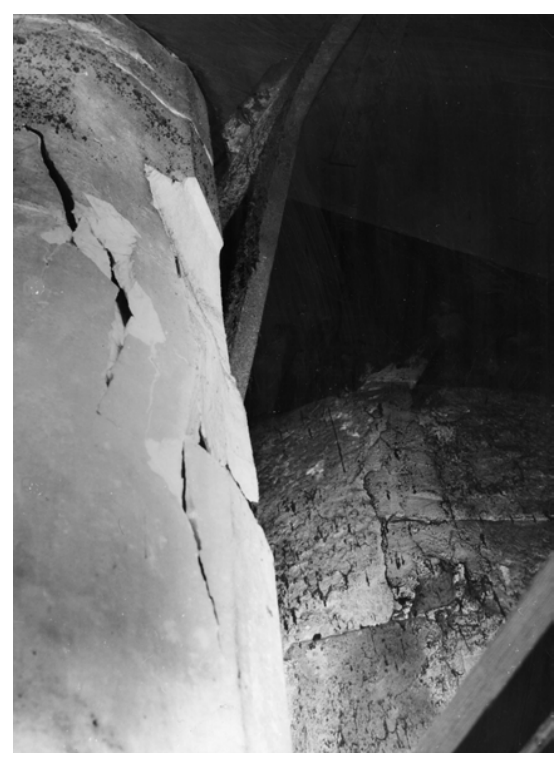

Fig. 1. Lesioni rinvenute sotto il pulpito del pilastro 84 (16 gennaio 1969). 
"fragilità" del monumento si è evidenziata nella seconda metà dell'Ottocento, quando sono stati eretti tre dei quattro "gugliotti" che agiscono in corrispondenza dei pilastri del tiburio (il cosiddetto Gugliotto Carelli era già esistente). Nonostante il loro peso relativamente modesto, la loro costruzione ha causato gravi dissesti, cui si è cercato di porre rimedio con interventi a volte discutibili.

Questa situazione non è esclusiva del Duomo di Milano, ma è condivisa da molti analoghi monumenti dell'epoca, i cui costruttori, indubbiamente notevolmente capaci, erano spesso anche piuttosto ardimentosi. Le grandi cattedrali, tuttavia, sono costruzioni estremamente massicce, essenzialmente soggette solo al loro peso, e una volta completata la loro costruzione (non tutte hanno raggiunto questo traguardo) possono resistere per secoli. Il Duomo di Milano non ha fatto eccezione e per quasi seicento anni ha superato indenne varie traversie, sopportando con danni relativamente limitati anche i bombardamenti della seconda guerra mondiale.

La stabilità di questi monumenti può tuttavia essere compromessa se intervengono fattori che alterino in maniera significativa le condizioni esterne. Nel caso del Duomo di Milano eventi di questo tipo si sono verificati negli anni cinquanta del secolo scorso e hanno alterato in maniera determinante le condizioni del terreno su cui poggiano le fondazioni dei pilastri, che come si è visto sarebbero oggi ritenute inadeguate. Il più dannoso è stato il marcato abbassamento della falda freatica, dovuto al notevole incremento nell'emungimento d'acqua che si è verificato nell'immediato dopoguerra, che ha diminuito considerevolmente la portanza del terreno, causando cedimenti differenziali della base dei piloni. Anche gli scavi per la costruzione della metropolitana e le vibrazioni indotte dal traffico di superficie e dai treni della metropolitana stessa hanno contribuito a compromettere la stabilità del monumento, con conseguenze che in breve tempo si sono fatte drammatiche.

Per fronteggiare la situazione l'ingegner Ferrari da Passano ha chiesto l'ausilio di competenze esterne e naturalmente il Politecnico di Milano ha svolto il ruolo più significativo in questo senso. Diversi docenti del Politecnico sono stati coinvolti e il loro contributo si è rivelato prezioso. In particolare, nel dicembre del 1965 il Consiglio di Amministrazione della Veneranda Fabbrica del Duomo ha nominato una commissione con il compito di affiancare l'ing. Ferrari da Passano per la definizione degli interventi che la gravità della situazione esigeva. Di questa facevano parte tre docenti del Politecnico, i professori Piero Locatelli, Leo Finzi (anche 
membri dell'Istituto Lombardo) e Guido Mangano, il professor Guido Oberti del Politecnico di Torino nonché un rappresentante della Fabbrica stessa. Data l'importanza del monumento e la gravità della situazione, alla fine del 1968 alla commissione è stata conferita maggiore ufficialità con nomina da parte del Prefetto, cui avrebbe dovuto rispondere del suo operato. In tale circostanza, essa è stata integrata con l'inserimento di rappresentanti di enti pubblici. Negli anni critici la commissione è stata presieduta dal professor Piero Locatelli, titolare della cattedra di Scienza delle Costruzioni del Politecnico e membro effettivo dell'Istituto Lombardo, che ha guidato l'operazione con ammirevole competenza e la ha condotta a termine con successo.

La commissione ha innanzi tutto provveduto alla messa in sicurezza del monumento: i pilastri del tiburio sono stati "incamiciati" da una corazza in calcestruzzo armato, mentre i pilastri secondari adiacenti sono stati rinforzati con putrelle verticali e cerchiature metalliche (Fig. 2). Si è
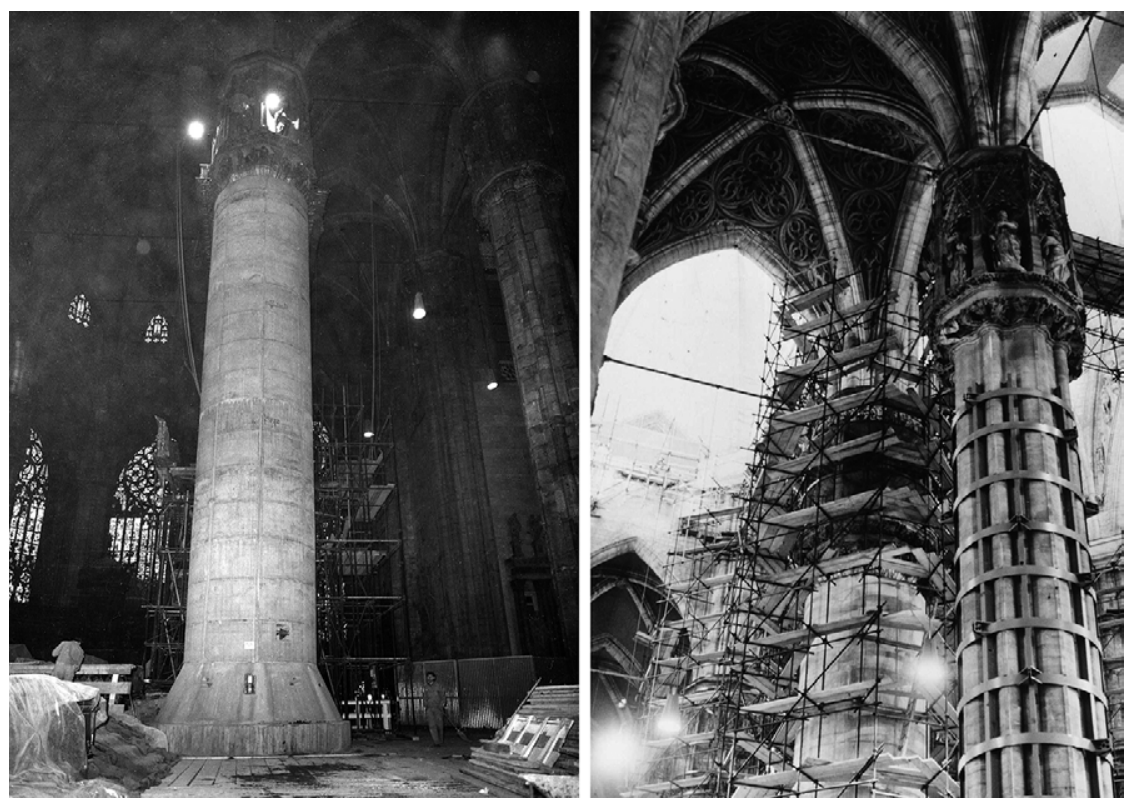

Fig. 2. Rinforzo provvisorio di un pilastro del tiburio (a sinistra) $e$ di un pilastro secondario adiacente. 
anche provveduto a ripristinare le catene del tiburio, tutte inefficaci (alcune fin dall'inizio). Sono stati poi presi alcuni provvedimenti "legislativi”. Per limitare gli effetti dell'abbassamento della falda, è stata ordinata la chiusura di tutti i pozzi di emungimento, molti dei quali abusivi, all'interno della cerchia dei navigli e le industrie operanti nella cintura periferica della città sono state obbligate a riciclare le acque prelevate. Sono state anche introdotte limitazioni al traffico veicolare di superficie e imposto il rallentamento dei treni della metropolitana in adiacenza della cattedrale, onde limitare le vibrazioni trasmesse al monumento. L'effetto delle vibrazioni, in realtà, si è rivelato non essere particolarmente critico e le limitazioni sono state rimosse dopo un certo tempo. Gli scavi per la realizzazione della metropolitana, che invece hanno certamente causato cedimenti forse significativi, erano all'epoca conclusi.

Tuttavia, l'aspetto saliente del lavoro della commissione ha riguardato la definizione degli interventi di consolidamento e restauro. Dopo aver esaminato e scartato diverse possibilità, tra cui il consolidamento chimico del terreno sottostante le fondazioni, si è optato per il rifacimento integrale dei pilastri del tiburio, operazione senza dubbio ardita e molto delicata. I pilastri erano (e sono tuttora) costituiti da una corona di conci di marmo di Candoglia che circonda un nucleo centrale di materiale meno nobile esteticamente e, nel caso specifico, molto povero dal punto di vista meccanico, sicché la funzione portante è affidata quasi interamente alla corona di marmo. L'intervento richiedeva di sostituire questa corona, operando praticamente concio per concio, senza compromettere in nessun momento la capacità portante del pilone, e si è svolto attraverso le fasi sintetizzate in Fig. 3, che vengono ora brevemente descritte.

Una lama rotante in acciaio diamantato rimuoveva una parte della camicia in calcestruzzo armato che costituiva il rinforzo provvisorio del pilastro. Da un settore della zona così liberata venivano tolti alcuni conci e si provvedeva a ripulire il vano creatosi e, nei limiti del possibile, a migliorare le condizioni del nucleo centrale. Nel frattempo, il cantiere marmisti produceva in tempo reale i nuovi conci, che venivano immediatamente introdotti manualmente e forzati in posizione "a martellate”, come indicato nell'ultima immagine. In questo modo, procedendo gradualmente dall'alto verso il basso, la corona in marmo di Candoglia è stata integralmente sostituita.

L'intervento ha avuto inizio nel gennaio del 1981 ed è stato intrapreso con il beneplacito dell'allora Arcivescovo di Milano, cardinale 

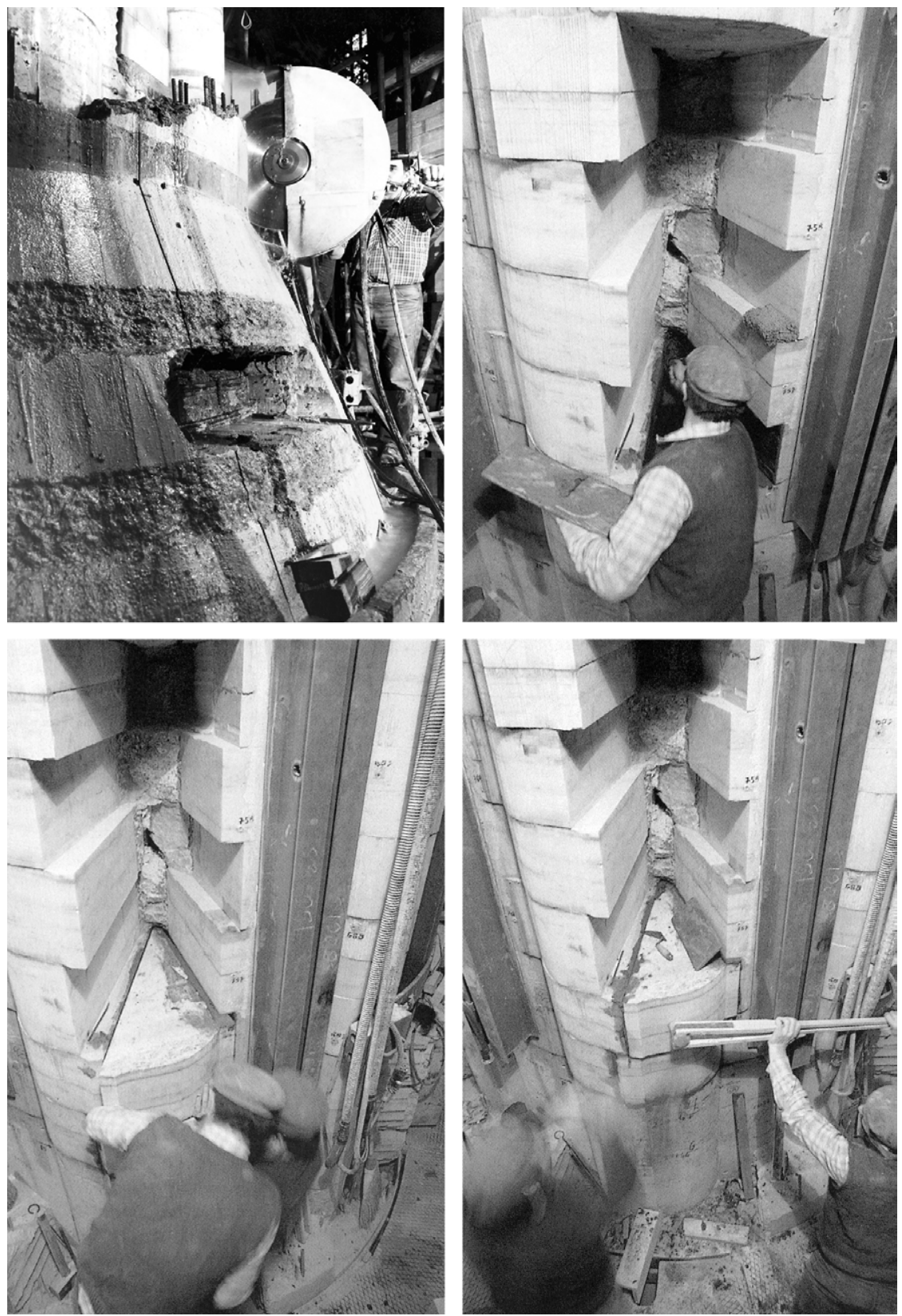

Fig. 3. Sostituzione di conci di marmo di un pilastro del tiburio. 
Carlo Maria Martini (membro effettivo dell'Istituto Lombardo) e ha avuto termine nel 1984. Il suo successo è testimoniato da quello che l'ingegner Ferrari da Passano chiamava "collaudo temporale": a trent'anni di distanza i cedimenti alla base dei pilastri (da allora costantemente tenuti sotto controllo) sono limitatissimi e i cedimenti differenziali praticamente nulli.

La conclusione dei lavori, tuttavia, non ha interrotto la collaborazione tra Politecnico e Fabbrica del Duomo. La Commissione Prefettizia, avendo espletato il suo compito, è stata sciolta, ma la Veneranda Fabbrica del Duomo ha chiesto e ottenuto che una nuova commissione venisse istituita, sia pure con compiti limitati alla consulenza e al coordinamento dei lavori che la cattedrale richiede permanentemente. Questa commissione, la cui componente tecnica è costituita in massima parte da docenti del Politecnico di Milano, è tuttora operante ed è intervenuta in diverse occasioni, anche se per problemi meno drammatici di quelli che ne hanno originariamente suggerito l'istituzione.

Il vero e proprio intervento di restauro ha avuto luogo nei primi anni ottanta del secolo scorso, ma la sua progettazione, che costituisce la parte più significativa del lavoro della commissione, presieduta negli anni critici dal prof. Piero Locatelli, è avvenuta nel decennio precedente, il che giustifica il titolo dato a questo lavoro, inteso a sottolineare il contributo del Politecnico di Milano.

\section{RINGRAZIAMENTO}

Le immagini qui riportate sono state fornite dalla Veneranda Fabbrica del Duomo di Milano, che si ringrazia per la sua cortesia. 